\title{
Improving the treatment of childhood acute lymphoblastic leukemia by optimizing the use of 70-year-old drugs
}

\section{William E. Evans}

Emeritus Faculty, Past-President and CEO, St. Jude Children's Research Hospital, Memphis, TN, USA

E-mail: WILLIAM E.EVANS - william.evans@stjude.org

doi:10.3324/haematol.2021.278967

$I^{n}$ the current issue of Haematologica, Larsen, Schmiegelow and colleagues ${ }^{1}$ have provided clear and convincing evidence that the addition of a relatively low dose of thioguanine $\left(\leq 12.5 \mathrm{mg} / \mathrm{m}^{2} /\right.$ day) can significantly increase the amount of thioguanine nucleotides incorporated into DNA (DNA-TG) of normal leukocytes in patients with ALL, when compared to treatment with only mercaptopurine in historical controls or in the same patients prior to the addition of low-dose thioguanine. As depicted in Figure 1, mercaptopurine requires intracellular metabolism by multiple enzymes to produce thioguanine nucleotides, whereas thioguanine is converted directly to thioguanine nucleotides. The deoxy thioguanine triphosphates are then available for incorporation into DNA, which is thought to be the principal mechanism of mercaptopurine's antileukemic effects.

Mercaptopurine is a mainstay of combination chemotherapy for the treatment of acute lymphoblastic leukemia (ALL), which is curative for over $90 \%$ of children and $\sim 70 \%$ of adults, whereas thioguanine is not widely used to treat ALL. Mercaptopurine was the first antileukemic agent for which pharmacogenomics was shown to be an important determinant of the optimal dosage, with those inheriting non-functional variants of thiopurine methyltransferase (TPMT) more likely to develop dose-limiting hematologic toxicity if treated with conventional doses of mercaptopurine $\left(75 \mathrm{mg} / \mathrm{m}^{2} / \mathrm{day}\right) ., 3$ In the 1990s, mercaptopurine became one of the first medications for which preemptive genotyping for common variants (in TPMT) were used to determine the optimal dosage, $e^{2,3}$ and 20 years later this strategy was expanded to include testing for inactivating variants in NUDT15 (nucleotide diphosphatase nudix hydrolase 15).,5 Nonfunctional alleles of TPMT are the primary determinants of mercaptopurine toxicity in people of European and African ancestry, whereas NUDT15 variants are the primary determinants in people of Asian and Native American ancestry. ${ }^{6}$ Patients who inherit two non-functional alleles for either of these enzymes must be treated with only $5-10 \%$ of the conventional dose of mercaptopurine to avoid toxicity, whereas for heterozygous patients it is recommended reducing the starting dose by about $50 \%{ }^{4}$ Even with these dose reductions, these enzymedeficient patients maintain higher average erythrocyte thioguanine nucleotide levels than homozygous wildtype patients treated with full doses of mercaptopurine and have comparable cure rates. It is unclear whether TPMT-deficient or heterozygous patients require supplemental doses of thioguanine to achieve DNA-TG in the target range and, if so, what dosage of supplemental thioguanine should be given.

As a complement to preemptive genotyping, monitoring the concentration of thioguanine nucleotides in erythrocytes is commonly used to identify patients who

accumulate excessive levels of thioguanine nucleotides or patients who have low thioguanine nucleotide levels due to non-compliance with daily oral mercaptopurine therapy. Although measuring thioguanine nucleotides in erythrocytes is clinically useful, this is not measuring the active drug in the target tissue (leukemia cells) nor active drug at the presumed site of action (thioguanine incorporated into the DNA). It will be important to determine in a large prospective clinical trial whether measuring thioguanine incorporated into DNA is indeed a better metric of mercaptopurine treatment than measuring thioguanine nucleotides in erythrocytes, because measuring thioguanine in DNA requires a more complex assay, which may not be widely available. Larsen et al. report that such a clinical trial (ALLTogether-1) is ongoing. It is interesting that in the current report, Larsen et al. did not find any correlation between median erythrocyte thioguanine nucleotides and median thioguaninine incorporated into leukocyte DNA (Online Supplementary Figure S6C in the article by Larsen et al. ${ }^{1}$ ).

Although it is not known how closely DNA incorporated into DNA of normal leukocytes reflects thioguanine incorporated into DNA of primary ALL cells in patients, it is reasonable to assume this is a better surrogate than measuring thioguanine nucleotides in the cytosol of erythrocytes, in part because only the trinucleotide is incorporated into DNA, whereas inactive mono- and di-phosphate nucleotides are measured in erythrocytes. Measuring thioguanine incorporated into the DNA of primary leukemia cells in patients would be the ideal metric, but this is not feasible because patients are generally in complete remission before mercaptopurine therapy is initiated and thus there are no leukemia cells to assess. It is also not known whether the incorporation of thioguanine into DNA of normal leukocytes has a uniform relation to thioguanine incorporated into DNA of leukemia cells of different molecular and lineage subtypes of ALL.

These limitations notwithstanding, measuring thioguanine incorporated into DNA (DNA-TG) of normal leukocytes offers a potential advance for optimizing mercaptopurine treatment of ALL. A major unknown is what level of thioguanine incorporation into DNA is indicative of optimal treatment with mercaptopurine, which will require assessment of the relation between thioguanine in leukocyte DNA and event-free survival in a large enough cohort of uniformly treated patients so that all relevant covariates can be included in a multivariate analysis.

It is also not known whether the level of increase in DNA-TG documented by Larsen et al. translates into an improvement in event-free survival, although Larsen et al. speculate that this could reduce the relapse hazard rate by as much as $59 \%$, based on their prior research reporting a relapse hazard ratio of 0.81 per $100 \mathrm{fmol} / \mu \mathrm{g}$ DNA increase (95\% confidence interval: 0.67-0.98; $P=0.029){ }^{7} \mathrm{~A}$ 


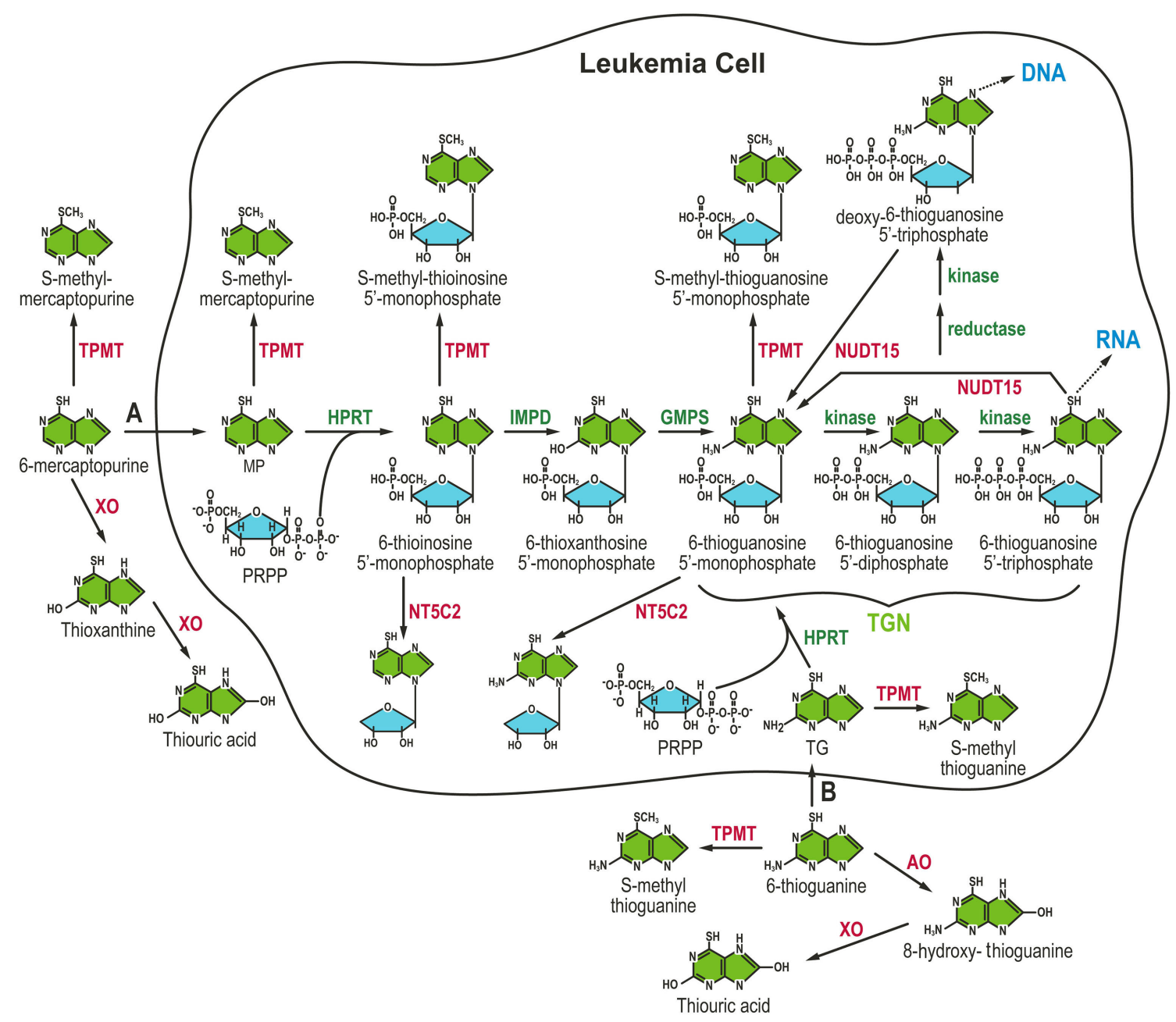

Figure 1. Schematic representation of the metabolism of mercaptopurine and thioguanine. (A, B) Metabolism of mercaptopurine (A) and thioguanine (B) and the enzymes involved hypoxanthine phosphoribosyltransferase (HPRT), inosine monophosphate dehydrogenase (IMPD), guanosine monophosphate synthase (GMPS), kinase, nucleoside kinases, thiopurine S-methyltransferase (TPMT), nucleotide diphosphatase nudix hydrolase 15 (NUDT15), 5'-nucleotidase, cytosolic II (NT5C2), xanthine oxidase (XO), aldehyde oxidase (AO) and phosphoribosyl pyrophosphate (PRPP). PRPP is a substrate in the reaction catalyzed by HPRT to form thiopurine nucleotides. The monophosphate of either the deoxy or ribonucleotide is formed by NUDT15, depending on the substrate. Enzymes shown in red catalyze inactivation of these thiopurine medications whereas those depicted in green are involved in activation to thioguanine nucleotides (TGN), which can be incorporated into DNA and RNA.

final issue that will need further study in a larger cohort of patients is whether the addition of this small dose of thioguanine is associated with additional toxicity during ALL therapy, as thioguanine has been associated with veno-occlusive disease in about $20 \%$ of children with $\mathrm{ALL},{ }^{8}$ and the risk with low-dose thioguanine when given concomitantly with mercaptopurine is unknown.

Nowadays, we are faced with the challenge of pushing the cure rate of childhood ALL beyond $90 \%$ while also improving the quality of life for those we cure. Improving the cure rate from $20 \%$ to $90 \%$ over the past six decades has been largely achieved by optimizing the use of conventional chemotherapy, such as mercaptopurine, not by the development of new antileukemic agents. Most of these improvements have been incremental in nature, but their cumulative effects have produced remarkable progress. $^{9,10}$ The work of Schmiegelow and colleagues ${ }^{1}$ may offer yet another small step toward maximizing the effects of medications that we have been using for many decades. Much hope and hype have been raised around the development of more targeted therapy for cancer, yet when available these targeted agents, such as tyrosine kinase inhibitors, are being added to and not replacing conventional chemotherapy in treating ALL. We would be wise not to abandon efforts to further improve the use of these older anticancer agents and avoid placing all our hope on so-called "targeted chemotherapy". And it should not go unnoticed that we continue to expand our knowledge of how best to use anticancer agents developed 70 years ago, suggesting that in the coming decades we may still be optimizing the use of both targeted and conventional chemotherapy as we work to push the ALL cure rate closer to $100 \%$.

\section{Disclosures}

No conflicts of interest to disclose.

\section{Acknowledgments}

I thank Mr. Josh Stokes for his many contributions to the preparation of the figure. 


\section{References}

1. Larsen RH Rank CU, Grell K, et al. Increments in DNA-thioguanine level during thiopurine-enhanced maintenance therapy of acute lymphoblastic leukemia. Haematologica. 2021;106(11)2824-2833.

2. Yates CR, Krynetski EY, Loennechen T, et al. Molecular diagnosis of thiopurine S-methyltransferase deficiency: genetic basis for azathioprine and mercaptopurine intolerance. Ann Intern Med. 1997;126(8):608-614.

3. Relling MV, Hancock ML, Rivera GK, et al. Mercaptopurine therapy intolerance and heterozygosity at the thiopurine S-methyltransferase gene locus. J Natl Cancer Inst. 1999;91(23):2001-2008.

4. Relling MV, Schwab M, Whirl-Carrillo M, et al. Clinical Pharmacogenetics Implementation Consortium guideline for thiopurine dosing based on TPMT and NUDT15 genotypes: 2018 update. Clin Pharmacol Ther. 2019;105(5):1095-1105.

5. Moriyama T, Nishii R, Perez-Andreu V, et al. NUDT15 polymorphisms alter thiopurine metabolism and hematopoietic toxicity. Nat Genet. 2016;48(4):367-373.
6. Yang JJ, Landier W, Yang W, et al. Inherited NUDT15 variant is a genetic determinant of mercaptopurine intolerance in children with acute lymphoblastic leukemia. J Clin Oncol. 2015;33(11):12351242 .

7. Nielsen SN, Grell K, Nersting J, et al. DNA-thioguanine nucleotide concentration and relapse-free survival during maintenance therapy of childhood acute lymphoblastic leukaemia (NOPHO ALL2008): a prospective substudy of a phase 3 trial. Lancet Oncol. 2017;18(4): 515-524.

8. Stork LC, Matloub Y, Broxson E, et al. Oral 6-mercaptopurine versus oral 6-thioguanine and veno-occlusive disease in children with standard-risk acute lymphoblastic leukemia: report of the Children's Oncology Group CCG-1952 clinical trial. Blood. 2010;115(14):27402748.

9. Pui CH, Yang JJ, Hunger SP, et al. Childhood acute lymphoblastic leukemia: progress through collaboration. J Clin Oncol. 2015;33(27): 2938-2948.

10. Pui CH, Evans WE. A 50-year journey to cure childhood acute lymphoblastic leukemia. Semin Hematol. 2013;50(3):185-196.

\section{Time to reconsider CD33 single nucleotide polymorphism in the response to gemtuzumab ozogamicin}

\section{Jatinder K. Lamba ${ }^{1}$ and Soheil Meshinchi ${ }^{2}$}

${ }^{1}$ Department of Pharmacotherapy and Translational Research, Center for Pharmacogenomics, College of Pharmacy, UF Health Cancer Center, University of Florida, Gainesville, FL and 'Division of Pediatric Hematology/Oncology, Fred Hutchinson Cancer Research Center, Seattle, WA, USA

E-mail: JATINDER K. LAMBA - jlamba@cop.ufl.edu

doi:10.3324/haematol.2021.279043

C D33 is a highly sought-after target in acute myeloid leukemia (AML), with gemtuzumab zogamicin (GO), a CD33-antibody conjugated to a DNA-damaging cytotoxin currently approved for the treatment of $\mathrm{CD}^{2} 3^{+}$adult and pediatric AML., ${ }^{1,2}$ The levels of expression of CD33 on the cell surface vary significantly between patients (up to 2 log-fold) and have been shown to be associated with disease characteristics as well as response to GO. ${ }^{3-5}$ However, as a biological threshold of CD33 expression that correlates with response to GO is lacking for incorporation into prospective trials to guide CD33-directed therapeutics, there is an urgent and unmet need to better define genomic variants that might predict response to GO.

It has recently been reported that there is a splicing single nucleotide polymorphism (SNP) in CD33, rs12459419 (C>T, Ala14Val), which results in skipping of exon 2 and thus loss of the most immunogenic domain of CD33 IgV. Given that the IgV domain is recognized by GO, this SNP holds great potential for predicting response to GO. Results from one of the largest studies to date (COGAAML $\left.0531^{\circ}\right)$ in children and young adults randomized to receive standard therapy with or without GO (GO arm, $\mathrm{n}=408$; no-GO arm, $\mathrm{n}=408$ ) indicated that there was a CD33 splicing SNP genotype-dependent clinical benefit from GO.? This study showed that the rs 12459419 C $>$ T change was significantly associated with CD33 cell surface levels $(P<0.001)$. With respect to clinical outcomes, in patients with the CC genotype ( $50 \%$ of patients) who expressed high levels of full-length CD33, addition of $\mathrm{GO}$ resulted in a significant reduction in relapse risk (by $~ 50 \%, P<0.001$ ), an improved disease-free survival and a trend to a better event-free survival in the whole cohort (disease-free survival, $P=0.004$; event-free survival, $P=0.055)$. In contrast, patients with the $\mathrm{CT} / \mathrm{TT}$ genotype had no benefit from the addition of $\mathrm{GO}$ to the standard no-GO therapy.' GO has been shown to improve outcomes in patients with favorable cytogenetics. ${ }^{8}$ Among low-risk patients in the COG-AAML0531 trial, a significant improvement in outcome with GO was observed in those with the CC genotype (relapse risk, $P<0.001$; disease-free survival, $P=0.001$; event-free survival, $P=0.001$; and overall survival, $P=0.014),{ }^{7}$ but not in patients with the CT/TT genotype. These results were consistent with the first report on the rs12459419 SNP which showed, albeit in a very small group of patients given $\mathrm{GO}$ after failing induction 1, an increase in minimal residual disease after $\mathrm{GO}$ treatment in patients with the TT genotype. ${ }^{9}$

These results raised hope for potentially personalizing GO treatment guided by germline SNP. However, initial attempts to validate these results in adult AML patients were apparently not successful, in two studies..$^{10,11}$ Some of the factors that might explain the inconsistencies from these studies are summarized in Table 1 along with key points from all the studies discussed in this article. There are a few points worth mentioning from the first study in 536 adult AML patients enrolled on MRC 15 and MRC $17 .{ }^{10}$ First, in contrast to the COG-AAML0531 study, in which there was a single randomization, these studies included multiple randomizations with patients receiving varying numbers of courses $(0,1$, or 2$)$ and doses of GO $\left(3 \mathrm{mg} / \mathrm{m}^{2}\right.$ or $6 \mathrm{mg} / \mathrm{m}^{2}$ ) and different induction and consolidation therapies of varying intensity, with the outcome analysis based only on GO exposure at initial induction. This randomization complexity also led to a lack of 